\title{
醏
}

Mercè Sala Rios*

Francisco X. Minguell Chillón ${ }^{* *}$

\section{POLÍTICA MONETARIA NO CONVENCIONAL Los programas de compra de activos del Banco Central Europeo}

La profundidad de la crisis económica ha planteado a la autoridad monetaria de los distintos países o áreas económicas unos retos sin precedentes a la hora de decidir las líneas a seguir en sus decisiones de política monetaria. Las medidas de carácter no convencional, especialmente las expansiones cuantitativas, han sido implementadas en diversas economías entre las que se encuentra la zona euro. El Banco Central Europeo inició, en el año 2015, un programa de compra de activos dentro de una estrategia de expansión cuantitativa inexplorada anteriormente. El objetivo de este estudio es analizar dichos programas de compras. Nos interesa conocer el porqué de su puesta en marcha, su evolución temporal y cuáles pueden acabar siendo, según los expertos, sus efectos sobre la economía de la zona euro.

Palabras clave: BCE, expansión cuantitativa, bonos, rentabilidad, facilidad de depósito.

Código JEL: E43, E58, H63, O52.

\section{Introducción}

La profundidad de la crisis iniciada a mediados de 2007 ha puesto en entredicho la efectividad de las principales políticas macroeconómicas y ha planteado unos retos a las autoridades hasta ahora poco imaginables. En el caso de la política monetaria se ha entrado en terrenos inexplorados que hacen muy atractivo conocer las consecuencias que puedan tener a largo plazo.

El Banco Central Europeo (BCE) no ha sido una excepción. En el año 2015 inició un

\footnotetext{
* Departamento de Economía Aplicada. Universidad de Lleida.

** Graduado en ADE. Universidad de Lleida.
}

Versión de julio de 2016 programa de compra de activos dentro de una estrategia de expansión cuantitativa (QE, por sus siglas en inglés), sin precedentes en la historia de la zona euro. Es una política no convencional que se tomó cuando la vía de los tipos de interés estaba casi agotada. El objetivo de este estudio es analizar dichos programas de compras. Nos interesa conocer el porqué de su puesta en marcha, su evolución temporal y cuáles pueden acabar siendo, según los expertos, sus efectos sobre la economía de la zona euro.

El trabajo se estructura en cinco apartados además de esta introducción. En el segundo se indican los principales motivos que llevaron a la implementación del programa de compras por parte del BCE. En el tercero se exponen $\triangleright$ 


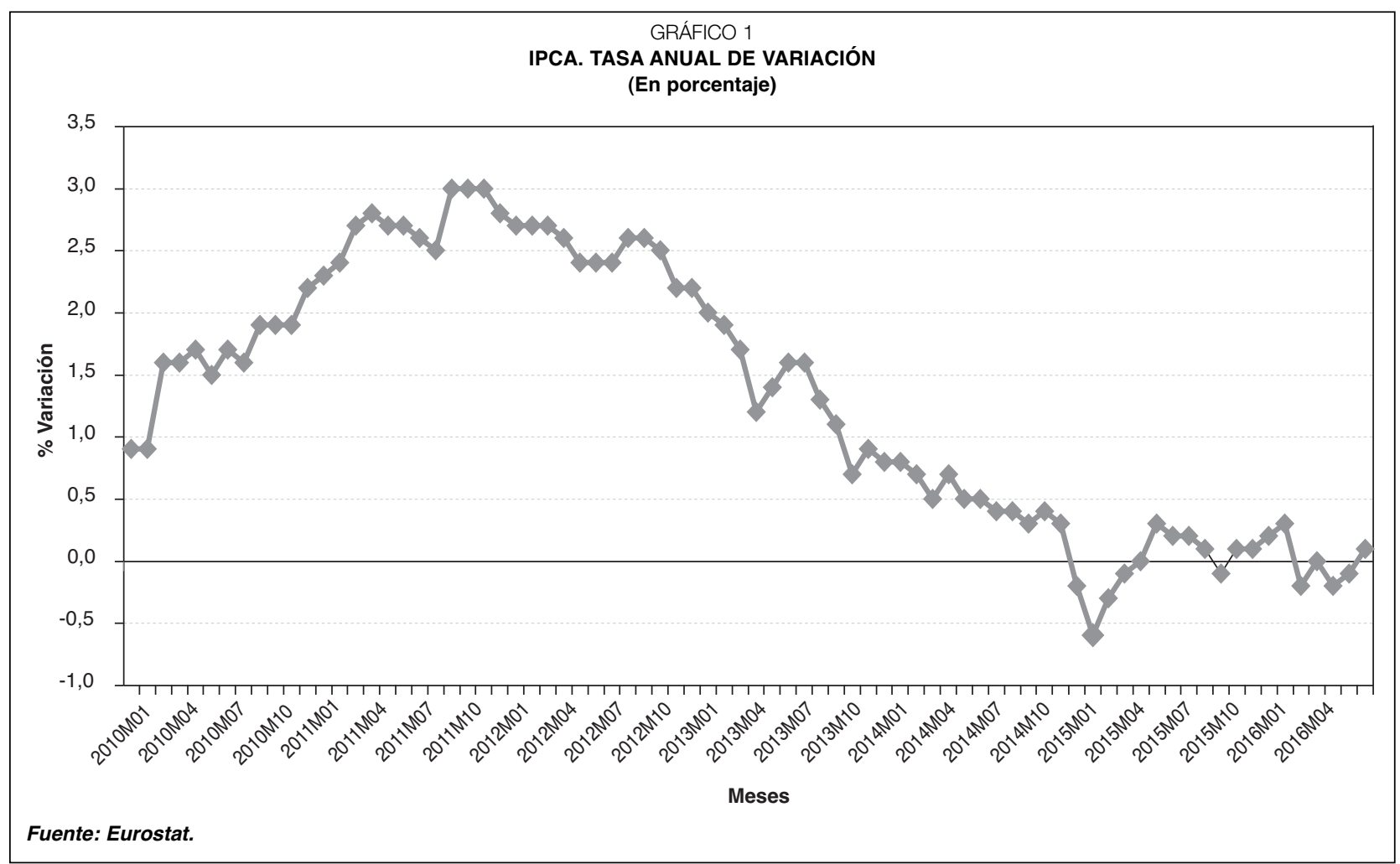

los subprogramas de compras puestos en marcha, así como los principales cambios que se han ido sucediendo con el paso del tiempo. El análisis de los posibles efectos, tanto positivos como negativos, del QE sobre las economías, así como su concreción en el caso de la zona euro, lo encontramos en el cuarto apartado. Unas conclusiones a modo de reflexiones finales cierran el trabajo.

\section{Quantitative easing (QE) en la eurozona: motivos de su implementación}

Desde el año 2012 hasta enero de 2015, que es cuando se anunció que se pondría en marcha un programa de compra de activos, la inflación en la eurozona fue disminuyendo para entrar en terreno negativo en el mes de diciembre de 2014 (Gráfico 1). Este deterioro de la inflación estuvo acompañado de unas tasas de crecimiento menores de las esperadas y de un aumento constante del peso de la deuda pública, especialmente de aquellas economías conocidas como periféricas.

La versión oficial para defender dicho programa de compra fue el riesgo de deflación. Sin embargo, la inflación subyacente durante los últimos meses del año 2014 se encontraba estabilizada alrededor del 0,7 por 100 y la del sector servicios, principal sector dentro de las economías desarrolladas, en el mes de diciembre de 2014 fue del 1,2 por 100. Por tanto, no parece que los datos de la inflación justificaran por sí solos la puesta en marcha de un programa de estas características.

Otros factores como el volumen de deuda pública, los tenedores de esa deuda, los déficits de muchos Estados y la cotización del euro respecto al dólar estaban también detrás de la decisión. En este sentido, cabe no olvidar que durante los años posteriores a la crisis de 2007 y, año tras año, muchos Estados $\triangleright$ 
comenzaron a tener déficits excesivos, lo que en muchos casos ha llevado a un crecimiento descontrolado de su endeudamiento, hasta el punto de entrar en un círculo vicioso que ha acabado provocando la solicitud de programas de rescate. Para cuadrar sus presupuestos o aproximarse a un cierto equilibrio presupuestario, los Estados necesitan tiempo que les permita realizar las reformas necesarias. EI BCE lo que pretende con su QE es rebajar, por un lado, el coste del endeudamiento y, por otro, dar estabilidad en los mercados mientras estas reformas se llevan a cabo, hechos a lo que se ha ido refiriendo de manera reiterada, en sus comparecencias públicas, el presidente del BCE, Mario Draghi. Por otra parte, se esperaba que el programa de compras actuara sobre una cotización de la moneda única que se encontraba en unos niveles considerados demasiado altos.

Otro motivo, que pudo influir a la hora de anunciar el $Q E$, deriva de un hecho que se había ido produciendo en el sistema financiero europeo durante los años previos. Con unos intereses cercanos al 0 por 100 , los bancos acudían en masa a las subastas de deuda pública, acumulando cantidades ingentes en sus balances. De esta forma, por un lado, los Gobiernos se aseguraban que buena parte de las emisiones quedaban cubiertas en unos momentos de mucha volatilidad y nerviosismo en los mercados. Por otro lado, para los bancos era un negocio mucho más seguro que el prestar a las familias y empresas dado que conseguían financiación con intereses extraordinariamente bajos por parte del BCE y lo invertían en deuda soberana que daba unos intereses más que aceptables y con menos riesgo. El hecho de reducir aún más las rentabilidades de la deuda pública, a través del QE, no dejaba de ser una medida para desincentivar su adquisición y obligarles a buscar otras fuentes para hacer negocio, como serían las familias y empresas.

En los meses previos a la decisión de puesta en funcionamiento del QE hubo mucho debate respecto de si el BCE podía ir tan lejos en política monetaria. Cabe no olvidar que los efectos de sus decisiones afectan de forma muy diferente a los distintos países miembros de la zona euro porque sus economías presentan amplias divergencias. Durante años se ha estado defendiendo la necesidad de una mayor integración como paso previo a una mejor capacidad de respuesta ante futuras crisis o shocks. Sin embargo, mayor integración implica compartir riesgos, lo que algunos países no están dispuestos a asumir. En los momentos más graves de la crisis de deuda de la eurozona se habló de emitir eurobonos, es decir, emisiones de deuda garantizada por la eurozona y no para un país en concreto. Esto habría provocado una transferencia de rentas entre países dado que algunos como España se financiaban al 5 por 100 y otros como Alemania al 1 por 100, por lo tanto, las emisiones de deuda conjuntas hubieran reducido notablemente el interés pagado por España pero se hubiera encarecido el de Alemania. Al final, por la oposición frontal de algunos países como Alemania, esta idea se fue abandonando. No es de extrañar entonces que, cuando se planteó la posible compra de deuda por parte del BCE, fuera precisamente Alemania una de las economías más beligerantes con el programa. El Bundesbank se ha erigido firme defensor de la ortodoxia europea y el QE es el máximo exponente de una medida monetaria no convencional. La compra de bonos públicos a gran escala es un punto de inflexión en $\triangle$ 
la hoja de servicios del BCE, nunca hasta ahora se había tomado una medida de estímulo tan agresiva.

En el mandato del Banco Central Europeo queda claro que éste no puede financiar Estados y debe centrarse en la estabilidad de los precios, por tanto, Alemania puso muchas objeciones a la realización del QE e incluso amenazó con llevar las posibles futuras medidas a los tribunales. Para poder poner en marcha el QE y que no hubiera impugnaciones por parte de algunos Estados, se decidió que sólo el 20 por 100 de los riesgos serían compartidos y que el 80 por 100 restante recaería en el banco central de cada país.

\section{Los programas de compra del BCE: plazos y evolución}

El QE tiene unos plazos prefijados que, sin embargo, pueden ir modificándose en función de la evolución y de las expectativas de algunos indicadores macroeconómicos como, de hecho, ya se ha producido. Para ver el QE en perspectiva, hay que acotar los principales acontecimientos que se han ido sucediendo.

El 22 de enero de 2015 Mario Draghi anunció en rueda de prensa que, a partir de marzo del mismo año, el BCE empezaría a aplicar el programa de compra de activos. La cuantía del programa era de 60.000 millones de euros mensuales y la vigencia del mismo se alargaba hasta septiembre de 2016. El total de la operación ascendía a 1,14 billones de euros.

Este programa está formado por tres subprogramas de compra diferenciados, que son los siguientes:

- Compras de valores del sector público (principalmente bonos soberanos y de Administraciones Públicas derivadas de los Estados).
- Compras de valores respaldados por activos (denominados Asset-Backed Securities o ABS).

- Compras de bonos garantizados del sector privado.

Por lo que respecta a la adquisición de valores del sector público, el BCE impone pocos límites. La institución comprará bonos de todos los países que forman parte de la eurozona, excepto de aquellos que formalmente se encuentran bajo un programa de rescate, como sería el caso de Grecia, que se regirán por unas normas particulares en función de la evolución de los compromisos pactados con los acreedores. Por tanto, también puede adquirir bonos que estén ofreciendo rentabilidades negativas, como sería el caso de los bonos alemanes a corto y medio plazo, aunque nunca podrán ofrecer rentabilidades negativas superiores a la facilidad de depósito, que actualmente se encuentra en terreno negativo. Prácticamente, el único punto donde el BCE fija una serie de límites es con respecto al volumen de las compras, puesto que se estipula que podrá adquirir más de un 25 por $100^{1}$ de una emisión siempre y cuando no sea un porcentaje superior al 33 por 100 del emisor. El BCE indicaba que el límite se establecía para evitar estar en una posición de poder de bloqueo ante una votación de una reestructuración de la deuda de un determinado país (BCE, 2015a).

En cuanto a los valores respaldados por activos (ABS), se compran en los mercados primarios y secundarios los tramos preferentes de bonos de titulización de activos así como aquellos tramos que, presentando una subordinación intermedia, disponen de garantías (senior y guaranteed mezzanine). A fin de $D$

${ }^{1}$ Ampliado hasta el 33 por 100 en la reunión del Consejo de Gobierno del BCE del 3 de septiembre de 2015. 
poder ser adquiridos en el marco del programa, los tramos senior de los ABS deben estar admitidos en el sistema de activos de garantía para las operaciones de crédito del Eurosistema, denominados en euros, haber sido emitidos en la zona del euro y tener una segunda mejor calificación crediticia, equivalente al menos al nivel 3 de la escala del Eurosistema, la correspondencia actual en términos de calificaciones sería BBB-/Baa3/ BBBI.

La compra de bonos garantizados era ya la tercera ronda que se aprobaba. La primera se puso en marcha en mayo de 2009 y se saldó con unas compras totales de 60.000 millones de euros. La segunda se lanzó en octubre de 2010 y aunque estaba dotada con 40.000 millones de euros, finalmente el BCE apenas empleó 16.000 millones. La tercera se anunció en septiembre 2014 y en 2015 quedó incorporada como un subprograma del programa de compras de activos.

Dado que los riesgos macroeconómicos por los que se puso en marcha el QE persistían y se empezaba a producir una serie de inestabilidades en los mercados, como son la ralentización de algunas economías emergentes y la brusca caída del precio del petróleo, el BCE decidió volver a actuar. El jueves 3 de diciembre de 2015 anunció nuevas medidas:

- El incremento temporal del QE, que pasa a estar vigente hasta marzo de 2017, lo que provoca que el total de la operación termine ascendiendo, como mínimo, a 1,5 billones de euros.

- Dentro de la franja negativa en la que ya estaba la facilidad de depósito se rebaja aún más situándola en el $-0,3$ por 100 , lo que permite comprar deuda que proporcione rentabilidades negativas hasta esta cifra.

- Los tipos de bonos que puede adquirir el programa de compra de activos incorporan emisiones regionales (como serían, en el caso español, las comunidades autónomas) y locales.
Si tenemos en cuenta la retórica utilizada en los días previos al anuncio de estas nuevas medidas, el BCE las consideraba insuficientes. Tal y como se confirmó más tarde públicamente, el responsable de poner freno a las mismas fue el Bundesbank, convirtiéndose de nuevo en la voz más discordante sobre la puesta en marcha de la expansión cuantitativa ${ }^{2}$.

Tres meses después, el 10 de marzo de 2016, el BCE volvió a aprobar nuevas medidas que incluyeron:

- El aumento de la cuantía mensual del QE que pasa de 60.000 millones de euros mensuales a 80.000 .

- La rebaja de la facilidad de depósito hasta el $-0,4$ por 100 desde el $-0,3$ por 100 anterior (recordemos que va ligada al tipo de emisiones que el BCE puede adquirir).

- En cuanto al límite en el volumen de las compras de un mismo emisor, éste aumenta del 33 hasta el 50 por 100.

- La inclusión directa de la compra de bonos corporativos Investment Grade (alta calidad).

También se anunció que se rebajaba el tipo de interés oficial al 0 por 100 y una nueva ronda de Long-term Refinancing Operation (LTRO). Las LTRO son préstamos a la banca a unos tipos de interés muy bajo y a largo plazo. Aunque no forman parte estrictamente del programa de compra de activos no está de más mencionarlas y ponerlas en perspectiva. Previamente a la puesta en marcha del QE, el BCE había estado desarrollando un amplio paquete de medidas de facilidad de crédito para evitar la caída de la oferta monetaria y las restricciones de liquidez que acompañan todos los procesos recesivos, las LTRO fueron las $\triangle$

2 El sistema rotativo de votos de los miembros del consejo provocó que el presidente del Bundesbank no tuviera voto en esta reunión. 
más destacables. Antes de la del 10 de marzo de 2016, el BCE ya había puesto en marcha dos rondas LTRO (una en diciembre de 2011 y otra en febrero de 2012) que ascendieron a la astronómica cifra de 1,018 billones de euros. Su objetivo principal era evitar el colapso de la banca y sustituir temporalmente el mercado interbancario que se encontraba prácticamente muerto.

La LTRO anunciada en 2016 presenta unas condiciones diferentes a las realizadas anteriormente. El tipo de interés es del 0 por 100 mejorable hasta llegar a la facilidad de depósito $(-0,4$ por 100) para aquellas entidades más activas en la concesión de créditos. Es decir, disfrutarán de este trato especial las entidades que demuestren que trasladan los beneficios de la LTRO a la economía real. Este hecho es importante porque es la primera vez que el BCE impone unas condiciones especiales en función de cómo se utilicen las medidas de facilidad crediticia que pone a disposición del sector financiero. El objetivo pasa a ser abrir de nuevo la financiación de la banca al sector privado.

Volviendo al programa del QE, cabe destacar que, desde su puesta en marcha y en menos de un año, ya ha sido modificado en dos ocasiones. Además, no sería de extrañar que en los próximos meses, en función de cómo se sucedan los acontecimientos, se vaya adaptando a las nuevas circunstancias. Al menos así lo ha querido dejar claro Mario Draghi al asegurar, tanto respecto a la duración como a la cuantía, que irá «hasta donde sea necesario" y en cualquier caso hasta que tengamos un alza sostenida de la inflación hacia el objetivo marcado por la institución.

Desde la reunión de marzo de 2016 no ha alterado ni en la cuantía ni en el tiempo el programa de compra de activos, simplemente se ha dedicado a hacer un seguimiento de la evolución del $\mathrm{QE}$, de los datos macroeconómicos y de las incertidumbres tanto económicas como políticas que se vislumbran en el horizonte. En referencia a las incertidumbres políticas, en la reunión del 21 de julio, el BCE analizó el impacto producido por el resultado del referéndum sobre la permanencia del Reino Unido en la Unión Europea. En ese sentido optó por no actuar prematuramente y esperar a la reunión de septiembre para decidir si debe tomar nuevas medidas o prorrogar las actuales. Los expertos opinan que optará por prorrogar el QE en caso de que se observe un deterioro de las expectativas de crecimiento europeas. Se considera que abrir la puerta a un precio oficial del dinero negativo es un paso que de momento el BCE no está dispuesto a dar y aumentar el importe de las compras es complicado dado que muchos tramos de bonos soberanos están en rentabilidades muy negativas $\mathrm{y}$, por tanto, no pueden incluirse en el programa según las normas vigentes. Ello sería posible si se rebajara aún más la tasa de facilidad de depósito, lo cual no es recomendable dada la delicada situación de la banca europea, especialmente en países como Italia ${ }^{3}$.

\section{Consecuencias de la implementación del Quantitative Easing}

Con este escenario, no podemos evitar preguntarnos si las medidas de estímulo han sido positivas, si directamente han sido ineficaces o incluso si a la larga pueden ser contraproducentes para las economías de la zona euro. En los próximos puntos nos planteamos $D$

\footnotetext{
3 Opiniones extraídas de una encuesta realizada por Bloomberg a 40 economistas sobre qué medidas tomará el BCE y cuándo.
} 
tanto cuáles son los efectos positivos como negativos que pueden derivar de un programa de compras de esta naturaleza. Estas ideas nos han de permitir finalizar realizando algunas reflexiones sobre lo que cabe esperar del QE implementado por el BCE.

\subsection{Posibles efectos positivos de un programa de compra de activos en la economía}

La profundidad de la recesión iniciada en 2007 acabó produciendo que, según la regla de Taylor (1993), para muchos países fuera recomendable situar sus tasas de interés en territorio negativo. Este hecho provocó que muchos bancos centrales comenzaran a estudiar seriamente la aplicación de medidas no convencionales. En Europa, uno de los pioneros en adoptar este tipo de medidas fue el Bank of England y, por tanto, es del que se dispone de más estudios sobre el programa y sus posibles efectos.

En el trabajo de Kapetanios et al. (2012) centrado en la economía del Reino Unido, se describen las dificultades de cuantificar el impacto de las medidas no convencionales. Sin embargo, mediante una serie de modelos econométricos, basados especialmente en los rendimientos de los bonos públicos, las tasas de inflación y el crecimiento del PIB, y tomando varios escenarios base, la conclusión a la que se llega es que, incluso en el escenario más pesimista, el QE tuvo un impacto positivo sobre el aumento de la inflación y la amortiguación de la caída del PIB de la economía del Reino Unido. Al mismo tiempo, la mayor demanda de bonos públicos presionó al alza su precio disminuyendo su carga financiera.

Según Demertzis y Wolff (2016) la adopción de un programa de compra de activos genera diversos factores positivos sobre la economía entre los que podemos destacar:

- El reequilibrio de carteras que conlleva la compra por parte del banco central de aquellos activos de menor riesgo presiona al alza sus precios y obliga a los inversores a tener que buscar inversiones más rentables y a la vez a asumir más riesgo.

- El banco central libera de los balances activos a cambio de liquidez, lo que es adecuado sobretodo en momentos de restricciones crediticias por culpa de un choque económico.

- La reducción de los costes financieros favorece la realización de nuevas inversiones al necesitar menores retornos de capital para ser rentables.

- La depreciación de la divisa nacional, que suele acompañar cualquier programa de estas características, favorece la competitividad de las exportaciones.

\subsection{Posibles efectos negativos de un programa de compra de activos en la economía}

Muchos economistas ven en algunos de los aspectos destacados en el punto anterior su lado negativo, calificando los efectos que pueden acabar produciendo sobre las economías como altamente peligrosos. Así, si bien destacábamos los efectos positivos, prácticamente incuestionables, de un programa de compra de activos sobre la disminución de las rentabilidades de los bonos públicos, esto es visto a largo plazo como un factor perjudicial por varias razones. En primer lugar porque puede llevar a que muchos operadores de mercado, que a menudo se mueven por motivos especulativos, quieran adquirir estos activos simplemente porque saben que el programa del $\triangleright$ 
banco central aumentará su precio de mercado. Con ello se corre el peligro de desencadenar una sobrevaloración que en el escenario más peligroso genere una burbuja especulativa. En segundo lugar, el supuesto reequilibrio de carteras que conlleva puede acabar implicando que muchas instituciones financieras acumulen en sus balances exceso de riesgos, precisamente uno de los motivos que llevó a la quiebra a grandes entidades bancarias globales y que fue el origen de la crisis financiera del año 2007.

La reducción de costes financieros, que suele acompañar un programa de estas características, puede contribuir a llevar adelante proyectos con escaso retorno económico. En un futuro, la vuelta a la «normalidad» en términos de tasas de interés es posible que los haga inviables y muchos acaben quebrando. La inestabilidad financiera que se generaría sería difícil de volver a reequilibrar con las mismas herramientas monetarias que han sido desarrolladas hasta ahora.

Además, Borio y Zabai (2016) señalan que la principal amenaza de un programa de compra de activos y de una política monetaria ultraexpansiva es que los Estados, familias y empresas opten por el sobreendeudamiento aprovechando los bajos tipos de interés. En el fondo sería contradictorio que aquellas medidas que el BCE puso en funcionamiento para paliar los efectos de la crisis que estalló en 2007 acaben produciendo los mismos efectos. Por eso no es de extrañar que a menudo se alerte sobre los riesgos que este tipo de medidas no convencionales pueden acabar comportando.

\subsection{Unas reflexiones sobre los efectos del $Q E$ implementado en la zona euro}

El discurso de Mario Draghi en sus últimas intervenciones busca ensalzar las virtudes y los resultados de un programa de compra de activos. Por ejemplo, durante su intervención del once de marzo de 2015 (BCE, 2015b), aseguraba que el impacto del $\mathrm{QE}$ ya era visible. Durante aquel discurso ponía de relieve los datos que especificamos a continuación.

Las tasas de los préstamos bancarios a las empresas comenzaron a declinar en el tercer trimestre del año 2014, las expectativas de inflación reaccionaron positivamente y en la zona euro los rendimientos de los bonos soberanos a largo plazo continuaron cayendo. La economía de la zona euro creció más de lo esperado en el cuarto trimestre de 2014 y el desempleo cayó a su nivel más bajo desde 2012. Aunque esto no puede atribuirse exclusivamente a la política monetaria expansiva del BCE y a las expectativas creadas por la deliberación durante los meses anteriores a su puesta en marcha, es evidente que ha ayudado. El presidente del BCE también ha venido insistiendo en que, aunque la inflación seguirá baja en los próximos meses, se espera que empiece a subir a partir de 2017, gracias a la política expansiva pero también animada por el incremento de los precios de la energía que la institución prevé que se producirá.

En la comparecencia de julio de 2016 el presidente de la autoridad monetaria detallaba que el PIB real de la zona euro había crecido un 0,6 por 100 intertrimestralmente. Un crecimiento que se debía al comportamiento de la demanda interna apoyada por la transmisión de las medidas de política monetaria. Las favorables condiciones de financiación se esgrimían como uno de los elementos que están estimulando la inversión (BCE, 2016).

Además, hay un convencimiento por parte de la institución de que en la zona euro este tipo de políticas monetarias se trasladan a la economía real, porque la financiación de la deuda corporativa se realiza principalmente a través de los bancos, a diferencia de los $D$ 


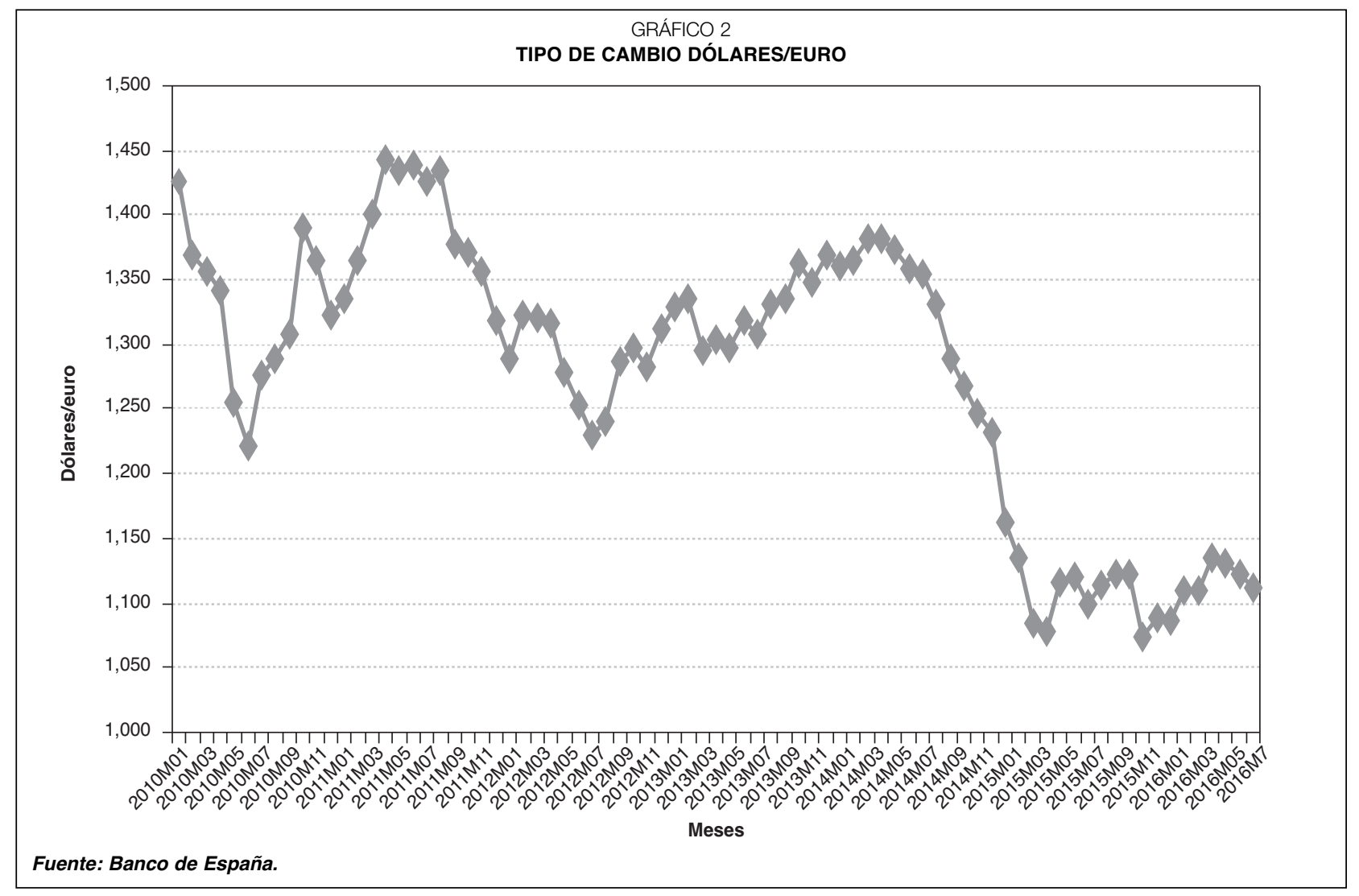

mercados de capitales de los Estados Unidos, por poner un ejemplo.

Mediante un modelo de equilibrio dinámico, Priftis y Vogel (2016) analizan los efectos del QE sobre la economía real. Los autores concluyen que el QE tendrá un efecto positivo sobre el crecimiento y los precios de la zona euro ya en la perspectiva del año 2016. Sin embargo, advierten que para la efectividad del programa será más débil si los bonos adquiridos provienen de inversores extranjeros. Por su parte, Claeys y Leandro (2016) opinan que uno de los efectos más visibles del QE es el que se ha producido sobre la cotización del euro. El tipo de cambio dólar/euro es ahora significativamente más débil en comparación con 2014, aunque ya había ido perdiendo valor debido, precisamente, a la especulación por parte de muchos operadores de la posible puesta en marcha del $\mathrm{QE}^{4}$ (Gráfico 2).

${ }_{4}$ Demertzis y Wolff (2016) esgrimen la normalización de la política monetaria en EEUU como otra causa de la evolución del tipo de cambio.
Por lo que se refiere a la amenaza de un sobreendeudamiento, aunque sea muy prematuro sacar conclusiones, los Gráficos 3 y 4 nos muestran que después de un año desde el inicio del QE no es palpable. Será interesante analizar cómo le afecta la ampliación del programa de compras y el trato especial que la nueva ronda LTRO da a las entidades que demuestren que trasladan los beneficios a la economía real.

Frente a todo lo anterior cabe contraponer que, como hemos visto en el Gráfico 1, desde el anuncio del QE en enero de 2015 la inflación continúa prácticamente estancada y, a pesar de ese 0,6 por 100 de crecimiento intertrimestral del mes de julio, las perspectivas de crecimiento anual no parecen haber mejorado mucho (Gráfico 5), y eso que ya se han inyectado más de 750.000 millones de euros en las economías de la zona euro.

En cuanto a la vigencia y extensión del programa de compra de activos, cuando se $D$ 

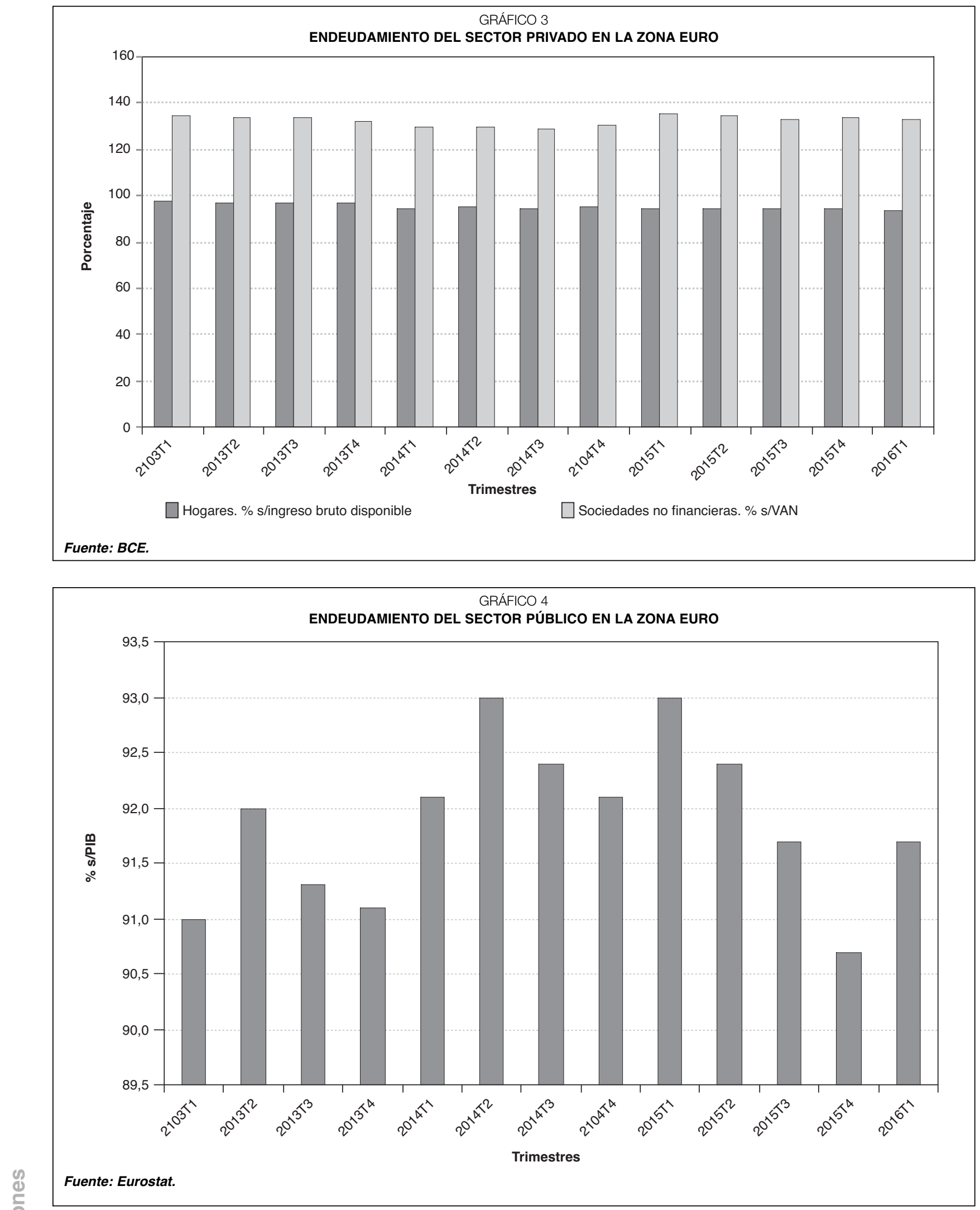

anunció su puesta en marcha muchos econo- iniciales, el programa no podría llevarse a camistas ya avisaron que, en las condiciones bo de manera completa hasta su vencimiento $\triangleright$ 


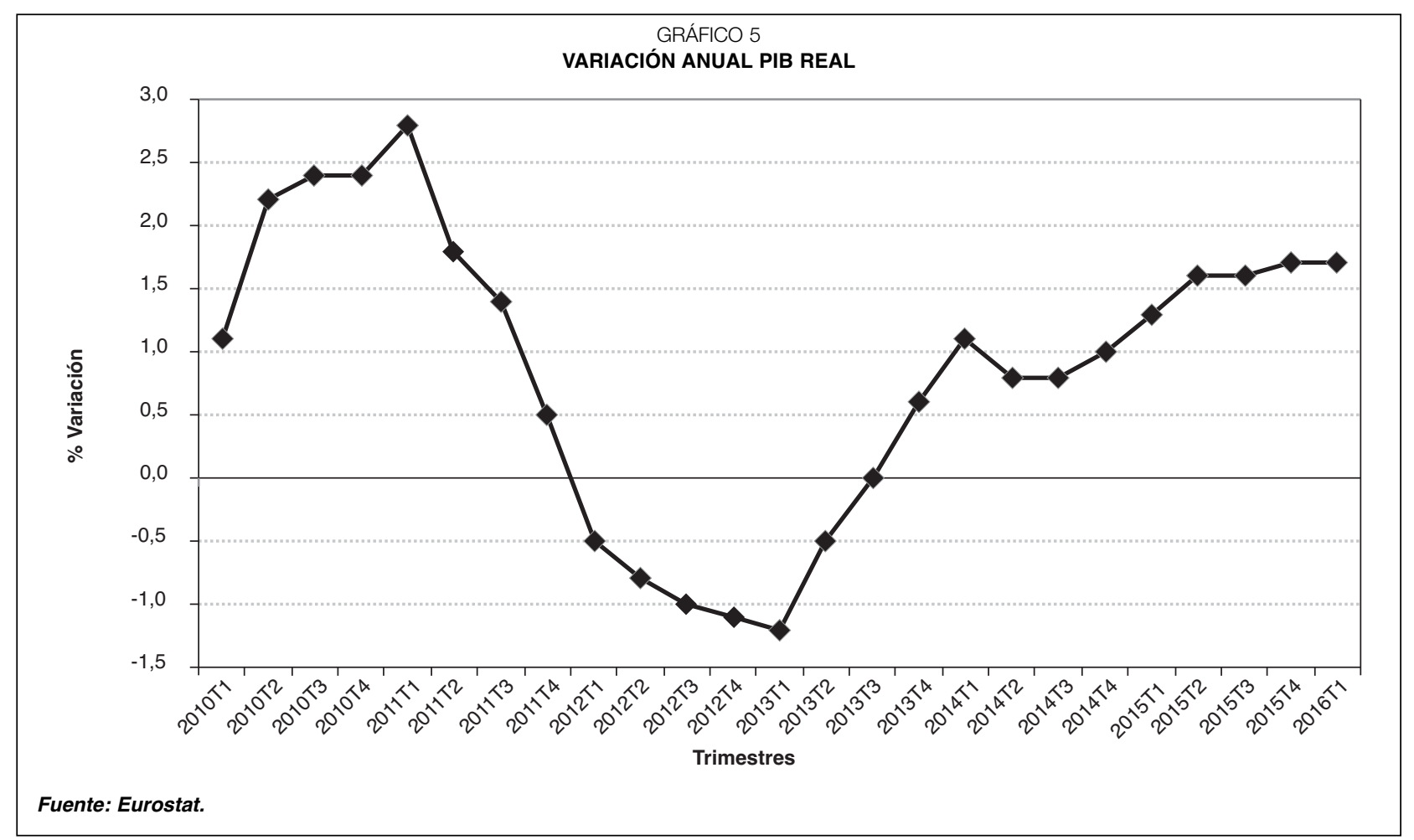

y que tendría que ser reformulado 5 . La eurozona la forman una serie de economías nacionales muy heterogéneas entre sí lo que implica que los activos que el BCE ha de adquirir tengan valoraciones muy diferentes. Con el estallido de la crisis de deuda soberana a finales de 2009, el diferencial de riesgo de los activos de aquellos países periféricos respecto de los de la Europa Central aumentó notablemente y si bien, con el tiempo, en la mayoría de ellos se ha producido una cierta convergencia (exceptuando casos como el de Grecia que aún se encuentra inmersa en un programa de asistencia financiera), los diferenciales siguen siendo importantes. Así, mientras países como España o Italia no habrían generado ningún problema a la hora de llevar a cabo el QE en las condiciones iniciales, otros como Holanda o Alemania no habrían permitido llevarlo a término dado sus rentabilidades negativas en los

5 En el trabajo de Claeys et al. (2015) se calculan, para los distintos países, los límites temporales del programa inicial de compra que en general, y sin tener en cuenta las ampliaciones que ha ido sufriendo, los autores situaban en marzo de 2017. bonos con vencimientos de menos de 10 años de duración (ver como ejemplo el Gráfico 6).

Desde su puesta en funcionamiento, y para poder continuar con las compras, tanto en cuanto a volumen como en cuanto a tipologías, las condiciones han tenido que ir modificándose. A fin de no incumplir los términos impuestos al principio de que sólo se puede adquirir aquellos bonos que no superen la facilidad de depósito, el BCE se ha visto obligado a rebajarla hasta el $-0,4$ por 100 . Profundizar en territorio negativo es peligroso puesto que este valor es al que tienen que hacer frente los diferentes bancos de la eurozona cuando depositan dinero en el BCE. El organismo es consciente de que cada vez que opta por rebajar la facilidad de depósito añade presión al sistema financiero europeo. Por lo tanto, es evidente que la estrategia de ir rebajando la facilidad de depósito para poder continuar con el QE lo limita de forma importante, tanto porque es una estrategia peligrosa por las consecuencias indirectas que conlleva como porque $D$ 


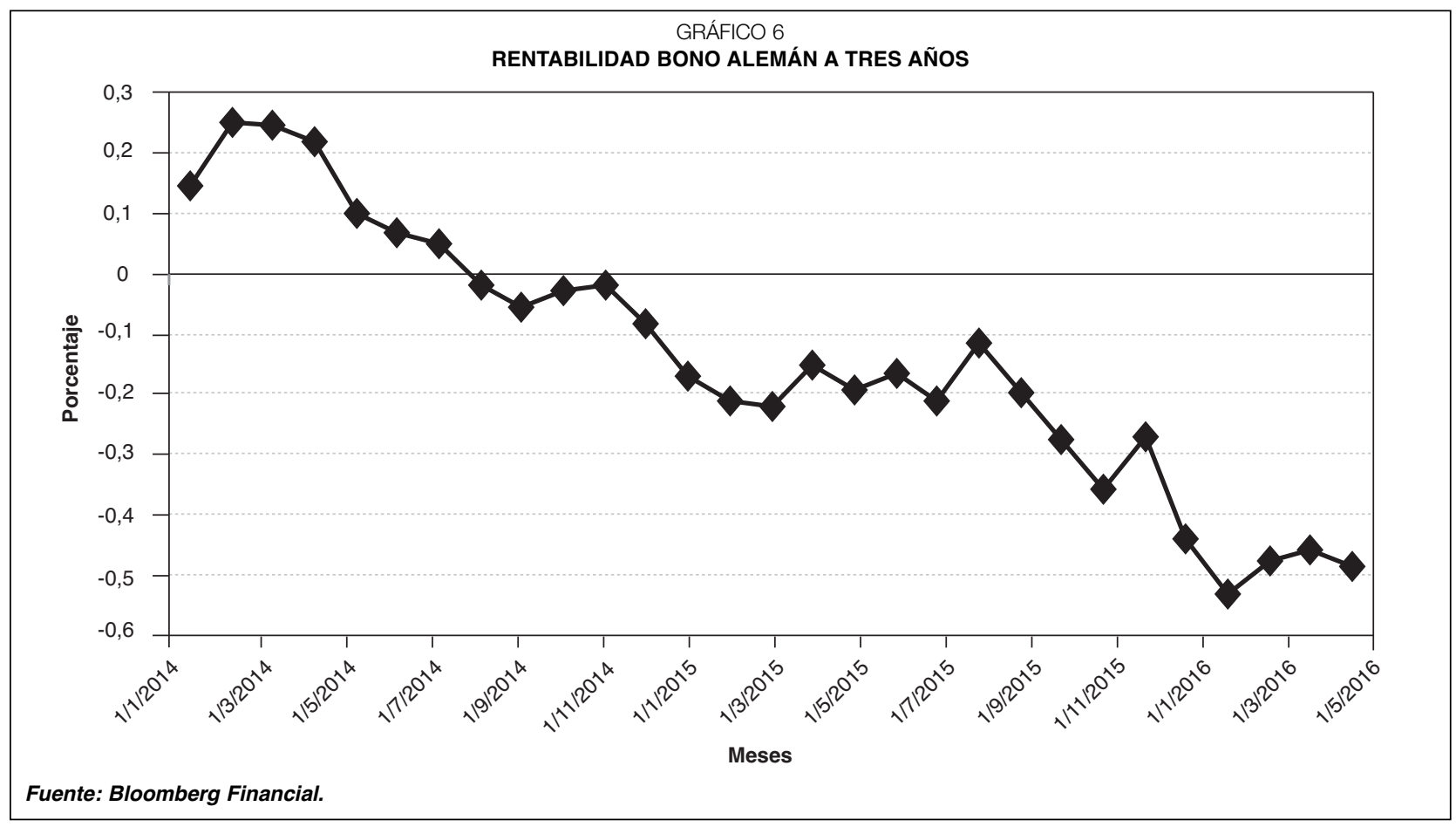

difícilmente será sostenible en el tiempo y menos en valores negativos como los actuales.

Las dudas en cuanto a su duración y extensión temporal radican también en la posibilidad de encontrar bonos que puedan entrar en el programa de compra, después incluso de la incorporación de los corporativos. El problema puede radicar más en las condiciones sobre la compra de una determinada emisión, impuesta en el propio programa, que por la escasez de títulos (Claeys y Leandro, 2016).

Todo esto en cuanto a las limitaciones a la hora de implementarlo en el tiempo, pero también es importante hacer referencia a aquellas limitaciones que se pueden producir desde el punto de vista de los efectos deseados. En este sentido cabe indicar que si bien el BCE persigue que los bancos acaben asumiendo más riesgos, por ejemplo en la concesión de préstamos, estos difícilmente lo harán si la presión reguladora es cada vez más elevada, tal como se está produciendo en el Eurosistema con las nuevas normativas aprobadas por el BCE (especialmente aquellas referentes a los requisitos de capital, dentro de los acuerdos de Basilea III). De hecho, Claeys y Darvas (2015) apuntan que los indicadores bancarios no sugieren un aumento sustancial en la asunción de riesgos. Por lo tanto, uno de los principales efectos positivos supuestamente producidos por el QE -el reequilibrio de carteras- quedaría en entredicho.

Por lo que se refiere a la caída de las rentabilidades de los bonos públicos, se observan resultados diferenciados ya que mientras algunos han continuado con su tendencia a ir reduciendo sus rentabilidades, otros se han mantenido relativamente estancados y en algunos incluso se han producido aumentos. Si se analiza este comportamiento de forma desagregada se observa que muchos de ellos coinciden con sus históricos y, por tanto, es más lógico pensar que es fruto de la heterogénea composición de las economías de la eurozona que no de consecuencias provocadas por la implementación del QE. 


\section{Conclusiones}

No resulta fácil aventurar cuáles van a ser las consecuencias de los programas de compra de activos del BCE. Como se desprende de lo expuesto en este trabajo hay varios elementos que así lo indican. En primer lugar vemos que los expertos en el tema no se ponen de acuerdo y lo que para algunos es un efecto positivo para otros a largo plazo puede acabar siendo contraproducente. En segundo lugar porque la realidad nos muestra que el BCE ha tenido que ir modificando y ampliando los programas dentro de unos períodos de tiempo muy cortos. Todo parece indicar que la línea de transmisión hacia la economía real de la política monetaria no convencional se está rompiendo continuamente. En tercer lugar, la complejidad de la crisis en una economía globalizada dificulta conocer los resultados y sus implicaciones.

Parecería evidente que programas de estas características fueran útiles ante un fuerte shock económico, dado que ayudan a introducir liquidez en el sistema, en un momento donde seguramente hay, y habrá, fuertes restricciones de la misma. Además, son actuaciones que ayudan a estabilizar el mercado de deuda, tanto pública como privada, en situaciones en las que es probable que la desconfianza pudiera llevar a empeorar la ya de por sí delicada situación de sus mercados.

Estamos entonces ante una política no convencional que debería llevarse a cabo en períodos de una fuerte crisis económica, en un espacio de tiempo muy concreto y sin retardos. Algunas de estas premisas no se dan en el actual programa de compra del BCE. Su inicio se produjo con bastante retraso y con unas condiciones de compra que han forzado a tener que ir ampliando el volumen de compra, cambiando las tipologías de activos que entran en el programa y rebajando los tipos oficiales hacia terrenos negativos poco recomendables desde la perspectiva de la economía real.

La incertidumbre sobre la efectividad de la política no convencional puede intuirse cuando el presidente del BCE viene indicando, en sus últimas comparecencias públicas, que si bien irá hasta donde sea necesario, la política monetaria por sí sola no siempre es capaz de conseguir los objetivos marcados. En su comparecencia de julio el presidente del BCE afirmaba, y no era la primera vez que lo hacía, que las políticas fiscales deberían apoyar la recuperación económica, pero sin dejar de observar los principios del Pacto de Estabilidad y Crecimiento (BCE, 2016). El problema radica en cómo articular ambos objetivos, es decir, ¿cómo puede la política fiscal favorecer el crecimiento económico sin perjudicar unas cuentas públicas y un nivel de deuda en el entorno de un área económica todavía afectada por una grave crisis de deuda?

Estamos, por tanto, ante un escenario complicado cara al futuro. Vemos por un lado que saltarse el principio de prudencia por parte del BCE no está teniendo los resultados deseados $\mathrm{y}$, según algunos expertos, puede acabar generando los mismos problemas que quería combatir. Y, por otro lado, sin haber salido de una crisis de la deuda y después de haber abogado durante mucho tiempo por políticas de austeridad, se pide a los Gobiernos que ayuden a la política monetaria. No cabe duda que para los analistas el futuro se presenta interesante pero para la economía real la perspectiva puede calificarse, al menos, como incierta. 


\section{Bibliografía}

[1] BCE (2015a). Implementation aspects of the public sector purchase programme (PSPP), 5 de marzo. Disponible en: http://www.ecb. europa.eu/ mopo/liq/html/pspp.en.html

[2] BCE (2015b). Comunicado preliminar, 5 de marzo. Disponible en: https://www.ecb. europa.eu/press/pressconf/2015/html/is1503 05.es.html

[3] BCE (2016). Comunicado preliminar a la conferencia de prensa, 21 de julio.

Disponible en: https://www.ecb.europa.eu/ press/pressconf/ 2016/html/is160721.es.html

[4] BORIO, C. y ZABAI, A. (2016). «Unconventional monetary policies: a re-appraisal». BIS Working Papers, ${ }^{\circ} 570$.

[5] CLAEYS, G.; LEANDRO, A. y MANDRA, A. (2015). «European Central Bank Quantitative Easing: the Detailed Manual», Bruegel Policy Contribution, $n^{\circ}$ 2015/02.

[6] CLAEYS, G. y DARVAS, Z. (2015). «The financial stability risks of ultra-loose monetary policy». Bruegel Policy Contribution, $n^{\circ}$ 2015/3.

[7] CLAEYS, G. y LEANDRO, A. (2016). «The European Central Bank's quantitative easing programme: limits and risks». Bruegel Policy Contribution, $\mathrm{n}^{\circ}$ 2016/4.

[8] DEMERTZIS, M. y WOLFF, G.B. (2016). «The effectiveness of the European Central Bank's asset purchase programme». Bruegel Policy Contribution, $\mathrm{n}^{\circ}$ 2106/10.

[9] KAPETANIOS, H.; MUMTAZ, G.; STEVENS, I. y THEODORIDIS, K. (2012). «Assessing the Economy-wide Effectsof Quantitative Easing", The Economic Journal, vol. 122, $\mathrm{n}^{\circ} 564$, pp. 316-347.

[10] PRIFTIS, R. y VOGEL, L. (2016). «The Portfolio Balance Mechanism and QE in the Euro Area». The Manchester School, doi: 10.1111/manc.12162. Version of Record online: 10 de junio de 2016.

[11] TAYLOR, J. B. (1993). «Discretion versus policy rules in practice». In: Carnegie-Rochester conference series on public policy, vol. 39, pp. 195-214. North-Holland. 